\title{
Stability and dynamical properties of the coexisting attractors of an external-cavity semiconductor laser
}

\author{
C. Masoller ${ }^{1}$ and N. B. Abraham ${ }^{2}$ \\ ${ }^{1}$ Instituto de Fisica, Facultad de Ciencias, Tristan Narvaja 1674, Montevideo, Uruguay \\ ${ }^{2}$ Department of Physics, Bryn Mawr College, 101 North Merion Avenue, Bryn Mawr, Pennsylvania 19010-2899
}

(Received 30 June 1997)

\begin{abstract}
Coexisting attractors, which arise from different external-cavity modes of the same longitudinal mode of the solitary laser, retain distinct stability properties, particularly when the laser is biased far above threshold and subjected to moderately strong optical feedback from a distant reflector. When the laser is modeled by the Lang and Kobayashi equations with additional gain nonlinearity, the dynamics is limited to external-cavity attractors that develop from the external-cavity modes which have a positive but not too large frequency shift with respect to the solitary laser emission frequency $\omega_{0}$. Although relaxation oscillations about these externalcavity modes are the first to become undamped as the feedback intensity increases, the attractors that arise from these modes remain stable over the largest range of feedback strengths. Stronger feedback destabilizes the individual attractors, creating new solutions which form from their ruins. At the beginning of the merging, the attractor ruins are not equally visited; the most visited ruins are those of the attractors last destabilized. We explore and explain these results by examining the dynamics of the laser when operating on a single externalcavity attractor. [S1050-2947(98)08101-3]
\end{abstract}

PACS number(s): 42.55.Px, 05.45. $+\mathrm{b}$, 42.65.Sf

\section{INTRODUCTION}

Semiconductor lasers are very sensitive to back reflections from any surface. Even very low levels of reflection light (power reflection rate $\approx 10^{-4}$ ) can destabilize singlemode operation of a semiconductor laser causing it to enter a regime characterized by high-intensity noise and a very broad linewidth. This behavior, termed "coherence collapse" [1], has been identified as a form of chaotic dynamics [2]. Both intrinsic interest in this type of nonlinear dynamics and practical applications of laser diodes have spurred a wide range of studies, many of which have been reviewed recently by Petermann [3] and van Tartwijk and Lenstra [4].

Some recent studies have focused on reducing or controlling the destabilizing effects of feedback [5-9]. Very weak feedback from short external cavities (typically, for cavities shorter than a few millimeters) can significantly reduce both the intensity noise and lasing linewidth [10]. The emission frequency can also be stabilized with phase-conjugate feedback [11]. Variations of the feedback within the range which leads to chaotic operation can be used to encrypt information $[12,13]$. Feedback into a broad-area laser can bias the selection of a particular lateral mode, though this is sensitive to very small variations of the external-cavity length $[14,15]$. Pulse-to-pulse jitter in spontaneously pulsing or externally switched laser diodes can be reduced with weak optical feedback, but the reduction is also extremely sensitive to small variations in the cavity length $[16,17]$.

Previous studies have considered short external cavities more than long external cavities, perhaps because for short external cavities coherence collapse can be more easily avoided and weak feedback can be used to improve frequency stability and reduce the laser intensity noise. However, many applications of integrated electro-optical systems involve optical feedback from more distant reflectors (typi- cally, from one to several meters). In this paper we study the transition to coherence collapse when the laser is biased relatively far above threshold, and when the feedback is from a distant reflector. The dynamics of a single-longitudinal-mode laser diode with optical feedback often has been described by the Lang and Kobayashi rate equations [18]. The external cavity is described by two parameters: the feedback parameter $\gamma$, which is proportional to the strength of the feedback, and the delay time $\tau$, which is proportional to the externalcavity length. Although the model does not include the multiple reflections in the external resonator, it describes observed laser behavior with high accuracy over a wide range of parameter values [2].

An important exception to the success of the Lang and Kobayashi model in describing experimentally observed dynamical phenomena is what has been termed the lowfrequency fluctuations (LFF) regime, which occurs for moderate feedback and when the laser is biased near the solitary laser threshold $\left(I<1.2-1.4 I_{\text {th }}\right)$. In this regime the laser intensity suddenly drops toward zero and then recovers gradually, only to drop out again after an apparently random delay. The physical mechanism behind LFF is still not fully understood, though several different explanations have been proposed. Mørk, Tromborg, and Christiansen [19] explained LFF as the result of bistability among the steady-state solutions introduced by the external cavity (the so-called external-cavity modes, ECMs). Hohl, van der Linden, and Roy [20] showed that spontaneous emission noise plays an important role in the nature and the statistics of the dropouts. Their experimental measurements agree with those of Henry and Kazarinov [21], in suggesting that spontaneous emission noise induces the dropout events. Sano [22] explained the dropouts as resulting from a switching among distinct regions of phase space. In this interpretation, the laser moves toward the mode with most gain, but since in the neighbor- 
hood of the maximum gain mode the modes and antimodes (i.e., unstable external-cavity modes) are very close, when the trajectory approaches an antimode, it is expelled into another region of the phase space, and then starts moving towards the maximum gain mode again $[23,24]$. As measurements of LFF more recently have become more accurate and as they have been made with higher-speed electronics, there is experimental evidence that the "power dropouts" are in the envelope of a series of short pulses, each lasting $100 \mathrm{ps}$ or less [24]. Similar pulses have been found in the Lang and Kobayashi model with high feedback [23,24]. Temporally resolved optical spectra reveal that there is enhanced power in several longitudinal modes during the power dropout $[25,26]$. The full significance of this faster dynamical behavior, not discussed in earlier LFF papers, and the possibility that it may excite (or be caused by the excitation of) more than one longitudinal mode will be an active area of future study. Recently, a different type of LFF have been found experimentally well above the laser threshold, with the signature of abrupt power increases instead of power dropouts [27].

When the laser is biased well above threshold ( $I$ $>1.4 I_{\mathrm{th}}$ ) the effects of spontaneous emission usually can be neglected. In the deterministic dynamics of the noise-free Lang and Kobayashi model, for increasing feedback more external-cavity modes appear and the previously existing modes become unstable though Hopf bifurcations (the previously damped relaxation oscillations, at a frequency similar to that of the solitary laser, become undamped) and quasiperiodic routes to chaos occur in the sequence of attractors that form near each mode as the feedback parameter is increased further [28].

Although the term coherence collapse is widely used whenever the laser linewidth broadens drastically from a few $\mathrm{MHz}$ to several $\mathrm{GHz}$, broadening does not necessarily imply chaotic dynamics. When the feedback is from a distant reflector, the round-trip time of the light in the external cavity, $\tau$, is much larger than the period, $\tau_{R}$, of the relaxation oscillations (of the laser intensity and the carrier number) in the solitary laser. In this case, the undamped periodic relaxation oscillations create what appears at low resolution to be a broadband spectrum without representing chaotic dynamics. Chaos in the attractor that evolves from a single externalcavity mode develops for higher feedback, while even higher feedback induces a deterministic merging of the ruins of several single ECM attractors to form a more complex, typically chaotic, attractor.

The dynamics has long and short time scales, as it involves fast evolution on the weakly unstable ruins of a particular ECM attractor interrupted by abrupt hopping into the ruins of a different attractor. This hopping gives both lowfrequency and broad band contributions to the spectrum but it differs from the low-frequency fluctuations which occur close to the solitary laser threshold. In the former the intensity fluctuations are more fully randomized, while the latter is characterized by random sudden drops in intensity followed by a deterministic stepwise buildup. In the LFF regime the evolution following a power dropout has a definite direction in phase space towards the external-cavity mode with maximum gain (the buildup process), while in the coherence collapsed regime the switching among attractors is more random. Given these distinctions, we will use the term coherence collapse to refer to the chaotic state in which there is merging of the basins of attraction which surround the individual external-cavity-mode solutions.

A similar transition from operation on the lowest linewidth mode to coherence collapse was recently studied by Hirono, Kurosaki, and Fukuda [29], who applied the injection locking model of Henry and Kazarinov [21]. In this alternative model the back reflected field is replaced by a constant field. The authors derive conditions on the strength of the feedback for the onset of coherence collapse, calculating when the damping of the relaxation oscillations vanishes. Our results differ from those of Hirono, Kurosaki, and Fukuda, since we treat the full time dependence of the back reflected field.

The remainder of this paper is organized as follows. Section II contains a brief description of the model. Through stability analysis and numerical solutions we show that the more stable of the external-cavity mode attractors which merge in the coherence collapsed state have frequencies slightly larger than the frequency of the solitary laser. In Sec. III we explain this result by examining the dynamics of the laser when it operates in a single ECM attractor. Section IV provides a summary and conclusions.

\section{DYNAMICS FOR MODERATE FEEDBACK AND LONG DELAY TIMES}

Single-longitudinal-mode semiconductor lasers can usually be successfully modeled with two dynamical equations, one for the evolution of the slowly varying complex electric field amplitude $E(t)$, and one for the evolution of the carrier population $N(t)$ (the electron-hole plasma). The Lang and Kobayashi equations are the usual laser rate equations plus a time-delayed term that takes into account the field reflected from the external mirror. Writing the intracavity electric field as $E(t) \exp \left\{i\left[\omega_{0} t+\phi(t)\right]\right\}$, where $E$ and $\phi$ are real and $\omega_{0}$ is the emission frequency of the solitary laser, the equations are

$$
\begin{gathered}
\frac{d E(t)}{d t}=\frac{1}{2}\left[G\left(N, E^{2}\right)-\frac{1}{\tau_{p}}\right] E(t) \\
+\gamma E(t-\tau) \cos \left[\omega_{0} \tau+\Delta \phi(t)\right] \\
\frac{d \phi(t)}{d t}=\frac{\alpha}{2}\left[G\left(N, E^{2}\right)-\frac{1}{\tau_{p}}\right]-\gamma \frac{E(t-\tau)}{E(t)} \sin \left[\omega_{0} \tau+\Delta \phi(t)\right],
\end{gathered}
$$

$$
\frac{d N(t)}{d t}=J-\frac{N(t)}{\tau_{s}}-G\left(N, E^{2}\right) E(t)^{2} .
$$

$E(t)$ is normalized so that $V_{c} E(t)^{2}$ is the total photon number in the solitary laser waveguide (where $V_{c}$ is the volume of the active region). $\Delta \phi(t)=\phi(t)-\phi(t-\tau)$ is the phase delay during the external-cavity round-trip time $\tau$. The feedback parameter $\gamma$ measures the strength of the light fed back into the laser cavity. Other parameters are $\tau_{s}$, the carrier lifetime; $\tau_{p}$, the photon lifetime; $G$, the gain per unit time $G=G_{N}\left(N-N_{0}\right)\left(1-\varepsilon E^{2}\right)$, where $G_{N}$ is the modal gain coefficient, $N_{0}$ is the carrier density at transparency, and $\varepsilon$ is the nonlinear gain coefficient which takes into account phe- 


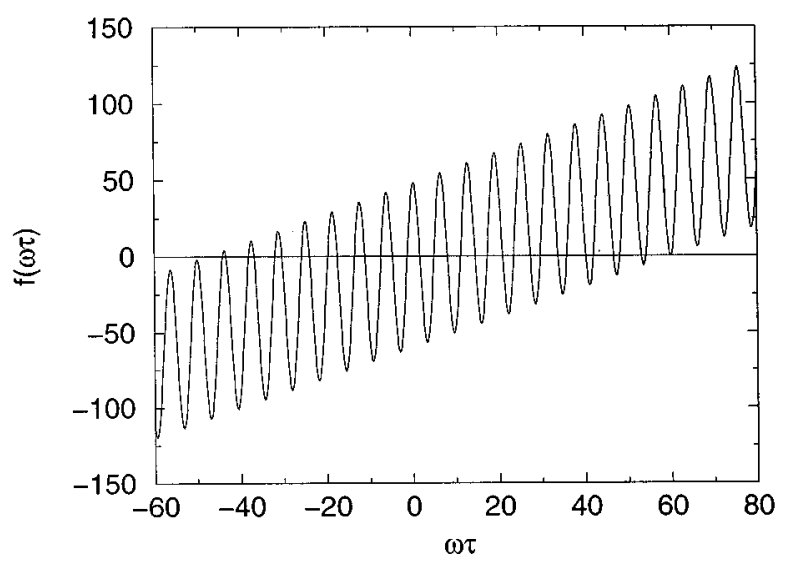

FIG. 1. Graphical solution of Eq. (4) for $\gamma=1.2 \times 10^{9} \mathrm{~s}^{-1}, \tau$ $=10 \mathrm{~ns}, \alpha=4.4$, and $\omega_{0} \tau=6$.

nomenologically the effects of carrier heating and spectral hole burning [30,31]. $\alpha$ is the linewidth enhancement factor $[32,33]$ and $J$ is the current density in carriers per unit volume and unit time. The frequently included Langevin noise terms that represent the effects of spontaneous recombination and spontaneous emission are omitted here, since these effects usually play a negligible role when the laser intensity is strong. We have chosen to retain the nonlinear gain $(\varepsilon \neq 0)$ since this more realistically models the experimental results, including more moderate pulsations (than those found with $\varepsilon=0$ ) which represent more confined trajectories in the phase space. There is qualitative similarity between the results for $\varepsilon \neq 0$ and $\varepsilon=0$ but for these numerical simulations we have opted for the more quantitatively realistic model. Of course any approximate nonlinearity has limited validity, but none of the solutions we report here leave that domain.

The stationary solutions of Eqs. (1)-(3) are called the external-cavity modes and can be written as $E(t)=E_{s, i}$, $\phi(t)=\left(\omega_{i}-\omega_{0}\right) t, N(t)=N_{s, i}$. The optical frequencies $\omega_{i}$ are the solutions of

$$
f(\omega \tau) \equiv \omega \tau-\omega_{0} \tau+\gamma \tau \sqrt{1+\alpha^{2}} \sin (\omega \tau+\arctan \alpha)=0 .
$$

The carrier density and field amplitude of a given ECM are found by solving

$$
G_{N}\left(N_{s, i}-N_{0}\right)\left(1-\varepsilon E_{s, i}^{2}\right)=1 / \tau_{p}-2 \gamma \cos \left(\omega_{i} \tau\right),
$$

and

$$
J-N_{s, i} / \tau_{s}-G_{N}\left(N_{s, i}-N_{0}\right)\left(1-\varepsilon E_{s, i}^{2}\right) E_{s, i}^{2}=0 .
$$

In Fig. 1 we show the graphical solution of Eq. (4), for $\gamma=1.2 \times 10^{9} \mathrm{~s}^{-1}, \tau=10 \mathrm{~ns}, \alpha=4.4$, and $\omega_{0} \tau=6 \mathrm{rad}$. Since the amplitude of the sine term in Eq. (4) depends on $\gamma$, and the period depends on $\tau$, the number of steady-state solutions increases as the value of either $\gamma$ or $\tau$ increases. The value of $C=\gamma \tau \sqrt{1+\alpha^{2}}$ determines this number. These solutions are called the external-cavity modes. For increasing feedback these modes are created in pairs after saddle-node bifurcations: for $C<1$ only one mode exists, which is the laser cavity mode minimally perturbed by the external cavity, while for $C>1$ there are $2 n+1$ modes. If
TABLE I. Laser parameters.

\begin{tabular}{lll}
\hline \hline Photon lifetime & $\tau_{p}$ & $1.4 \mathrm{ps}$ \\
Carrier lifetime & $\tau_{s}$ & $1 \mathrm{~ns}$ \\
Linewidth enhancement factor & $\alpha$ & 4.4 \\
Threshold current & $J_{\text {th }}$ & $2.08 \times 10^{33} \mathrm{~m}^{-3} \mathrm{~s}^{-1}$ \\
Injected current & $J$ & $2 J_{\text {th }}$ \\
Modal gain coefficient & $G_{N}$ & $8.39 \times 10^{-13} \mathrm{~m}^{3} \mathrm{~s}^{-1}$ \\
Carrier density at transparency & $N_{0}$ & $1.23 \times 10^{24} \mathrm{~m}^{3}$ \\
Gain saturation parameter & $\varepsilon$ & $2 \times 10^{-24} \mathrm{~m}^{3}$ \\
Feedback phase & $\omega_{0} \tau$ & $6 \mathrm{rad}$ \\
\hline \hline
\end{tabular}

$$
\left.\frac{d \omega_{0}}{d \omega}\right|_{\omega_{i}}=1+\gamma \sqrt{1+\alpha^{2}} \cos \left(\omega_{i} \tau+\arctan \alpha\right)<0
$$

the solution is intrinsically unstable (a saddle point), and is called an antimode [2] [the antimodes are modes for which the slope of $f(\omega)$ is negative]. In Fig. 1 we see that for $\gamma$ $=1.2 \times 10^{9} \mathrm{~s}^{-1}$, and $\tau=10 \mathrm{~ns}$ there are 17 modes and 16 antimodes.

Equations (1)-(3) were integrated with a fourth-order Runge-Kutta method with an integration step $\Delta t=0.01 \mathrm{~ns}$ and using the parameters given in Table I (the same as in Ref. [34], where it was found that the visibility in interferometric coherence measurement of a laser diode within the coherence collapsed regime is accurately reproduced by the Lang and Kobayashi model). The external-cavity parameters $(\gamma$ and $\tau$ ) are the free parameters of our study. The feedback is varied from zero to well above the value for which attractor merging begins, and the delay time is kept greater than 3 ns, corresponding to relatively large external cavities $\left(L_{\text {ext }}>0.5 \mathrm{~m}\right)$.

For increasing feedback each stable ECM undergoes a Hopf bifurcation to periodic oscillations followed by a quasiperiodic route to chaos. The time-dependent solutions are often localized in phase space near the steady-state solutions (ECMs), indicating separated basins of attraction. The two frequencies that appear in the route to chaos are, to a good approximation, the same for all the attractors in the basins of attraction around the different modes, and are approximately the relaxation oscillation frequency of the solitary laser $\left[f_{\mathrm{RO}}=\sqrt{G_{N}\left(J-J_{\mathrm{th}}\right)} / 2 \pi\right.$, where $J_{\text {th }}$ is the threshold current density] and the external-cavity-mode spacing frequency $\left(f_{\text {ext }}\right)$, which is slightly lower than $1 / \tau$ [2].

Since the ECMs have optical frequencies $\omega_{i}$ shifted positively and negatively with respect to $\omega_{0}$, it is not surprising that the different chaotic attractors to which they evolve have different mean optical frequencies. When the chaotic attractors merge in the coherence collapsed regime, these frequency differences show up in the different characteristic rates of phase accumulation. A typical trajectory in the merged regime is shown in Fig. 2 (the circles indicate the positions of the destabilized external-cavity modes, and the crosses, the positions of the antimodes). We plot the trajectory in the plane formed by $\Delta \phi(t)+\omega_{0} \tau$ and the normalized electric field $E(t) / E_{\text {sol }}$ (where $E_{\text {sol }}$ is the field amplitude of the solitary laser). The steady-state value of $\Delta \phi(t)+\omega_{0} \tau$ when the laser operates in the external-cavity mode $i$ is $\omega_{i} \tau$ 


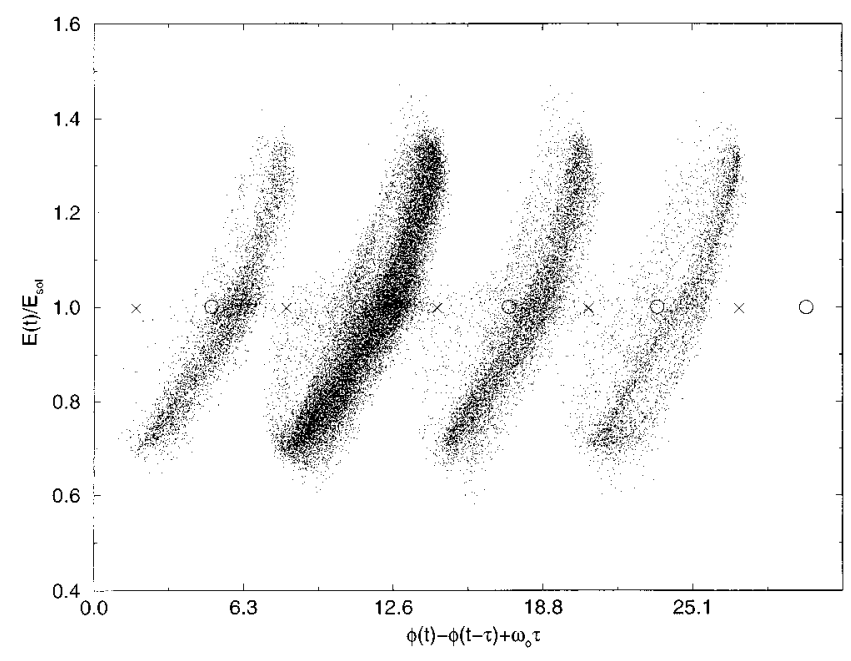

FIG. 2. Trajectory at the beginning of coherence collapse $(\gamma$ $=3 \times 10^{9} \mathrm{~s}^{-1}, \tau=10 \mathrm{~ns}$ ). Eighty round-trips in the external cavity are shown. The evolution occurs in the attractor ruins of four external-cavity modes $(i=0,1,2$, and 3$)$.

(if $i$ is not too large, the values of $\omega_{i} \tau$ are separated by approximately $2 \pi$, see Fig. 1 ).

Since $f_{\mathrm{RO}}$ and $f_{\mathrm{ext}}$ appear in the attractors that develop from any of the modes (at least, for large external cavities for which $f_{\text {ext }} \ll f_{\text {RO }}$ ), for moderate feedback the optical spectrum of the laser will show peaks spaced at the harmonics (and linear combinations) of $f_{\mathrm{RO}}$ and $f_{\mathrm{ext}}$, regardless of whether the laser is operating on an attractor near a single ECM or if it is switching among attractors near different ECMs. This makes it hard to distinguish the underlying nature of the dynamics just from the optical spectrum or the intensity power spectrum.

The optical spectrum of a multimode or pulsing laser diode is asymmetric under many conditions [35]. This is due principally to the $\alpha$ factor, which couples the modulus and phase fluctuations of the complex electric field. However, we find that at the beginning of attractor merging, the optical spectrum is not only asymmetric in the heights of the peaks spaced by $f_{\mathrm{RO}}$ and $f_{\text {ext }}$ but usually the main peak is not at the operating frequency of the solitary laser, $\omega_{0}$, but is shifted positively. We show in Fig. 3 the optical spectrum for four different feedback levels. Figure 3(a) has the same parameter values as Fig. 2 and corresponds to the beginning of attractor merging (for these parameter values, $f_{\mathrm{RO}}=6.65 \mathrm{GHz}$ and $f_{\text {ext }}=0.1 \mathrm{GHz}$ ). These spectra have obvious asymmetries about the major peaks, and two characteristic spacings between peaks. The central peak is shifted by one or more multiples of the ECM frequency spacing towards positive frequency, which is especially noticeable in Fig. 3(a). For increasing feedback the frequency shift gradually disappears [Fig. 3(d)].

The shift in the optical spectrum comes from the tendency of the laser to operate in attractors which originate from ECMs with $\omega_{i} \geqslant \omega_{0}$, in spite of the fact that the ECMs are located symmetrically in frequency with respect to $\omega_{0}$ (as shown in Fig. 1). In Fig. 2, which corresponds to the same parameter values as Fig. 3(a), the laser switches among the attractor ruins originated from ECMs $0 \leqslant i \leqslant 3$.

For increasing feedback the first ECM attractors to be-
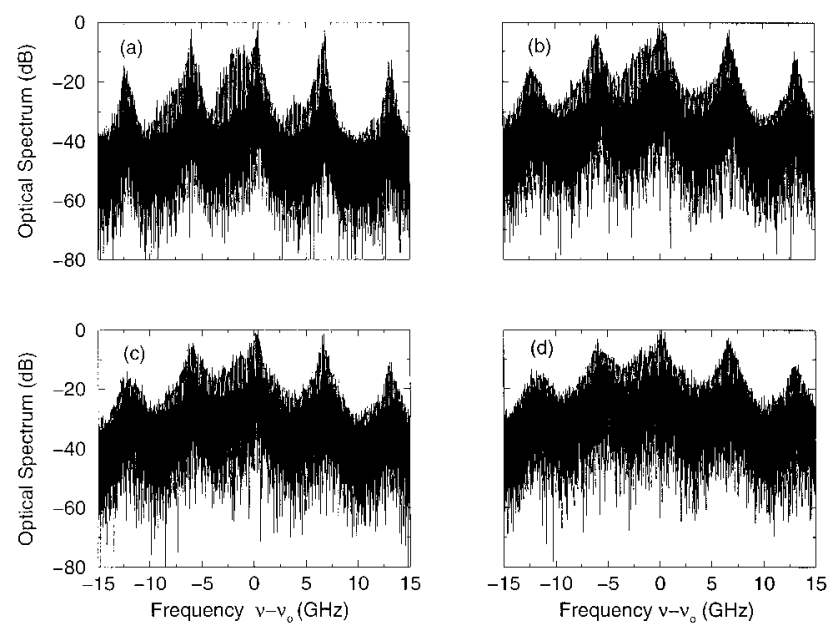

FIG. 3. Optical spectra at the beginning of coherence collapse $\tau=10 \mathrm{~ns}$ and (a) $\gamma=3$, (b) $\gamma=3.5$, (c) $\gamma=4.0$, and (d) $\gamma=4.5$ $\left(10^{9} \mathrm{~s}^{-1}\right)$. The spectrum was calculated with 262144 points sampled with $\Delta t=0.01 \mathrm{~ns}$, which span 262 round-trips in the external cavity. The parameters are the same as for Fig. 2 .

come "attractor ruins", are those with the largest round-trip phase difference with respect to the solitary laser (i.e., with mode number $i \gg 0$ ). Trajectories with initial conditions that place them near these unstable attractors will evolve, after a transient, to one of the remaining attractors near ECMs with low positive mode number (typically, $0<i<5$ ), which are stable over a large feedback range. The ECMs with $i \ll 0$, which are often stable fixed points, have very tiny basins of attraction; trajectories with initial conditions in the vicinity of these modes will also evolve to the attractors near ECMs with low, positive mode number. When the last of these ECM attractors has lost its stability, merging begins.

The merged attractors are not equally visited, as seen by the different densities in the zones of Fig. 2. The attractor hopping dynamics can be characterized by the probability of visiting the different ECM attractor ruins as follows. We recorded the trajectory for a long time and computed its Poincare section with the plane $E=E_{\text {sol }}$. The probability residency in the different attractor ruins was then estimated from the density of intersection points. Figures 4(a) and 4(b) show the Poincaré section and the corresponding probability residency, for the same parameters as for Fig. 2. The integration time is much longer than for the data shown in Fig. 2. The trajectory briefly visits the attractor ruins of the ECMs $i=-1$ and 4 , and spends most of its time in the ruins of ECMs $i=1$ and 2. On the horizontal axis of Fig. 4(b) we have indicated with a circle the location of the destabilized external-cavity modes, and with a cross the most probable values (which are shifted to the right).

In order to obtain good statistics, it is important to compute the trajectory for a long time, since at the beginning of attractor merging the jumps are not very frequent, and the trajectory spends a long time in one attractor before switching to another. The accuracy of the probability distributions was checked by calculating them over different trajectories (with different initial conditions), typically by beginning in different attractor ruins, including those not visited in the merged attractors (that have not merged yet). After a transient in which the trajectory evolved towards the merged 


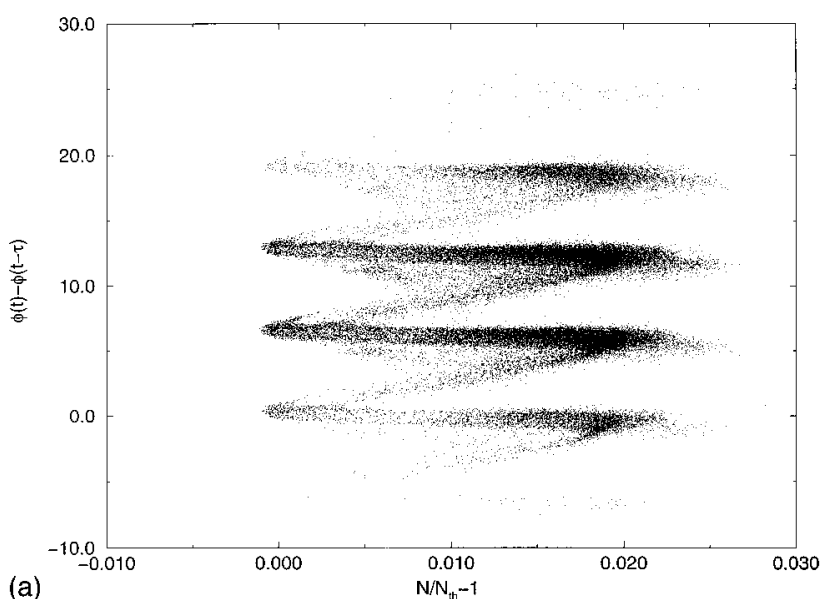

(a)

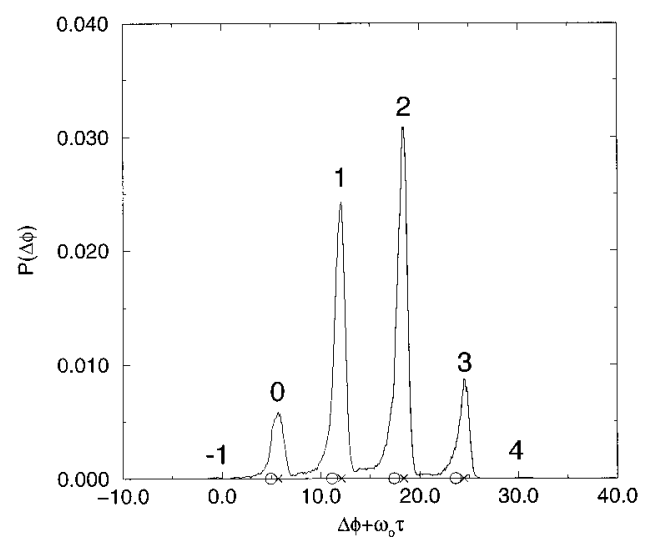

(b)

FIG. 4. (a) Poincaré map for the same parameters as Fig. 2. 100000 points are shown. Since the oscillation period $\tau_{R}$ is $0.15 \mathrm{~ns}$, in a round-trip time there are approximately 67 intersections of the Poincaré plane. Therefore 100000 points correspond to approximately 1500 round-trips. (b) Probability distribution obtained from the Poincaré map of (a).

attractors, the shape of the probability distribution remained unchanged in time.

Figure 5 shows that the number of visited ECM attractor ruins increases and the relative residences shift as the feed-

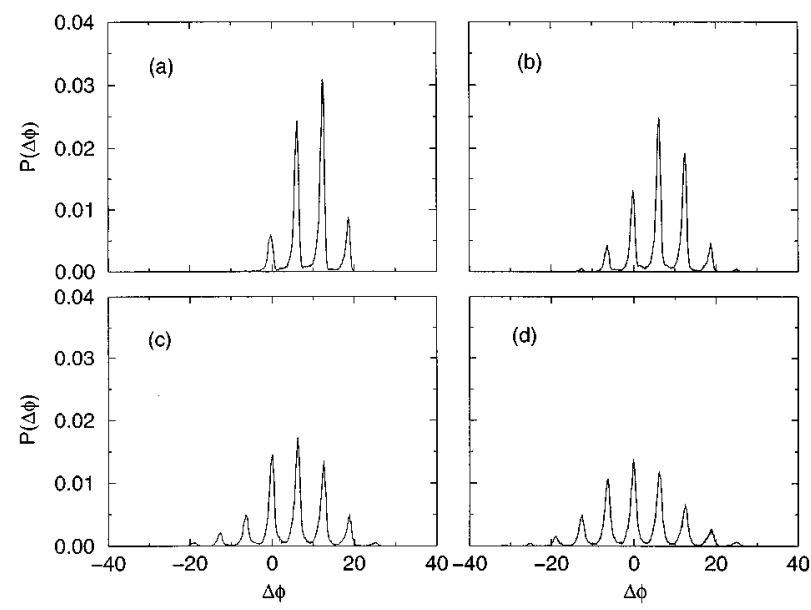

FIG. 5. Probability distribution for four different feedback levels within the coherence collapse regime. $\tau=10 \mathrm{~ns}$, and (a) $\gamma=3.0$, (b) $\gamma=3.5$, (c) $\gamma=4.0$, and (d) $\gamma=4.5 \times 10^{9} \mathrm{~s}^{-1}$.

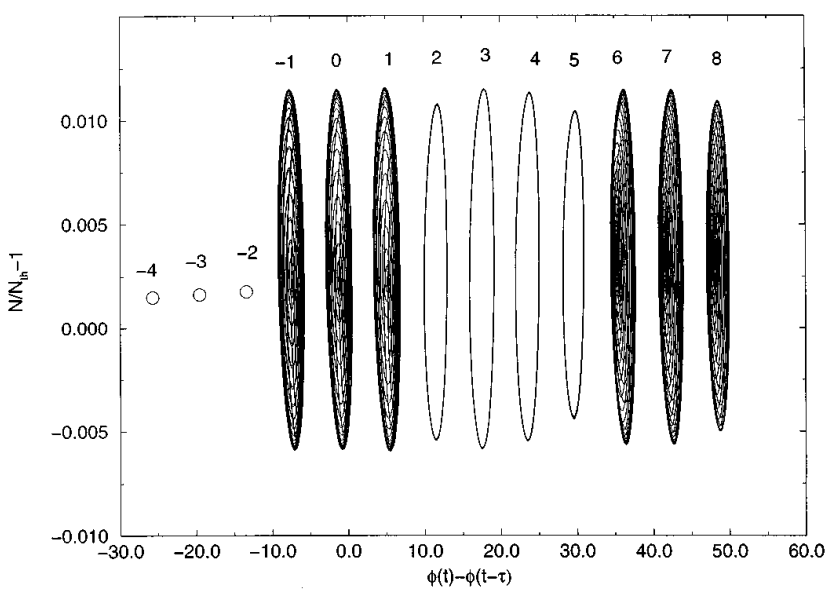

FIG. 6. Coexisting attractors for the same parameter values as Fig. 1. The external-cavity modes $i \leqslant-2$ are fixed points (only three are shown, represented with circles). From the external-cavity modes $-1 \leqslant i \leqslant 1$ and $6 \leqslant i \leqslant 8$ quasiperiodic tori develop, while from the external-cavity modes $2 \leqslant i \leqslant 5$ limit cycles develop.

back changes (the parameters are the same as in Fig. 3). In Fig. 5(a) four attractors have merged, the probability distribution is clearly asymmetric, and the most visited ECM attractor is $i=2$, centered at an optical frequency larger than the solitary laser frequency. For increasing feedback [Figs. $5(\mathrm{~b})-5(\mathrm{~d})]$, more neighboring attractors join the merged global attractor. The jumps become more frequent, and as the number of attractors involved increases, the probability distribution becomes symmetric and more nearly centered on $\omega_{0}$. Consequently, the frequency shift in the optical spectrum disappears for increasing feedback [Fig. 3(d)].

Therefore we conclude that for moderately strong feedback from a distant external reflector the laser tends to operate on attractors near the external-cavity modes with a positive but not too large frequency shift with respect to the solitary laser state. The modes (and the attractors to which they evolve) with large frequency shifts are highly unstable (those with a positive shift) or have very narrow basins of attraction (those with a negative shift). The laser is very rarely observed to operate in the vicinity of these modes. In the next section we examine the dynamics of the laser operating on an attractor near a single ECM.

\section{DYNAMICS IN SINGLE ECM ATTRACTORS}

In Fig. 6 we show the coexisting attractors for the parameter values used in Fig. 2 but at a lower feedback level (the feedback is the same as in Fig. 1, which is below the onset of attractor merging). In the simulations the initial conditions were taken to be the unstable steady-state solutions given by Eqs. (4)-(6), and transient evolution during the first roundtrips of the external cavity was discarded (typically 300 round-trips).

In all for these conditions there are seven fixed points, four limit cycles, and six quasiperiodic tori. The externalcavity modes which exist with mode number $i \leqslant-2$ are all stable, though only three are shown. The attractors which have developed from modes $-1 \leqslant i \leqslant 1$ and $6 \leqslant i \leqslant 8$ are tori, and the attractors which have developed from modes $2 \leqslant i$ $\leqslant 5$ are limit cycles. 


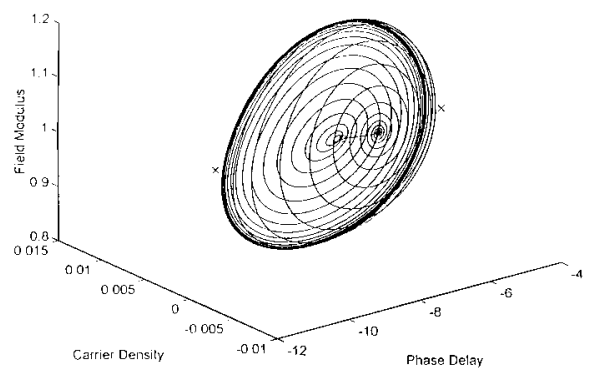

(a)
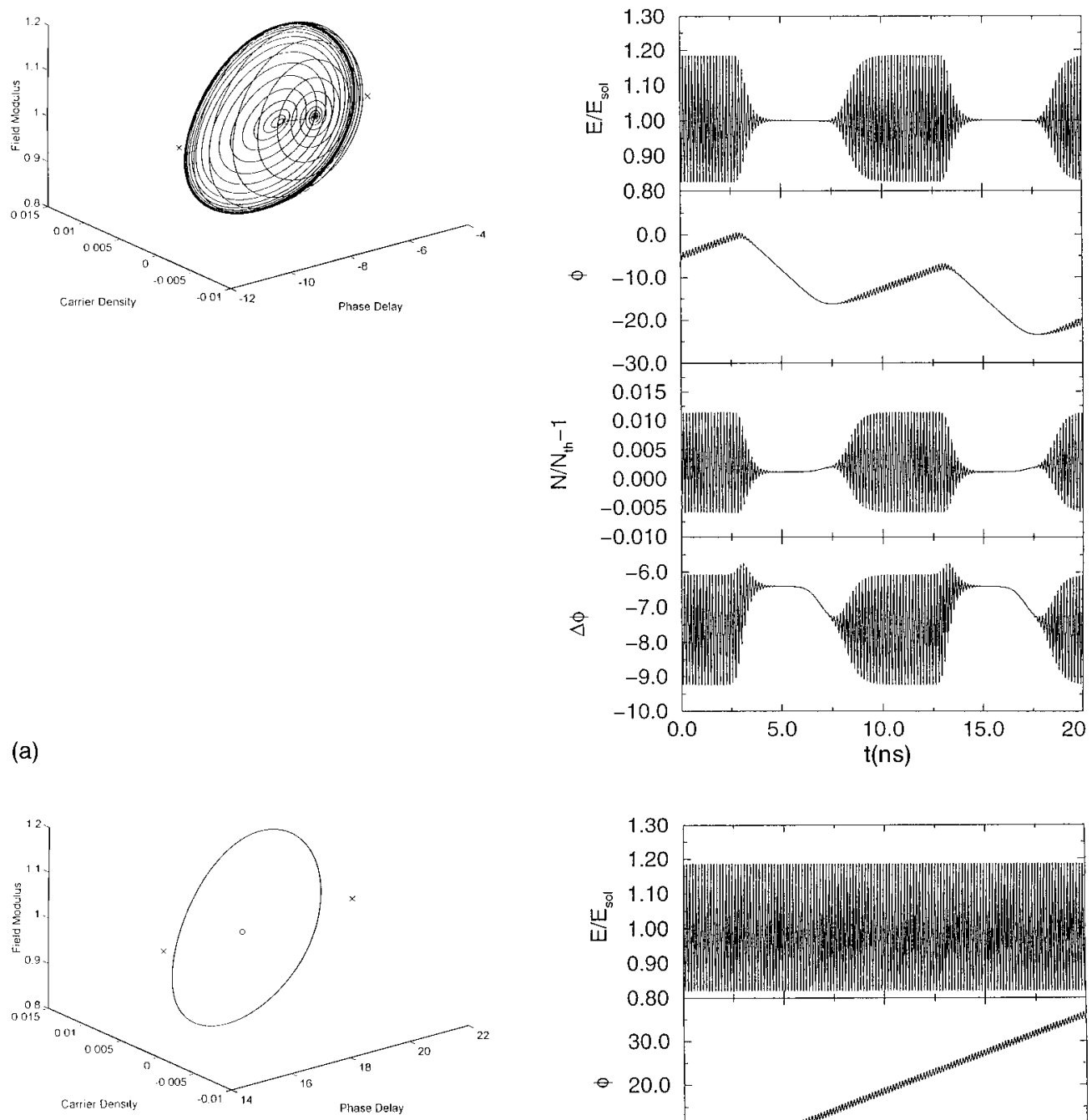

(b)

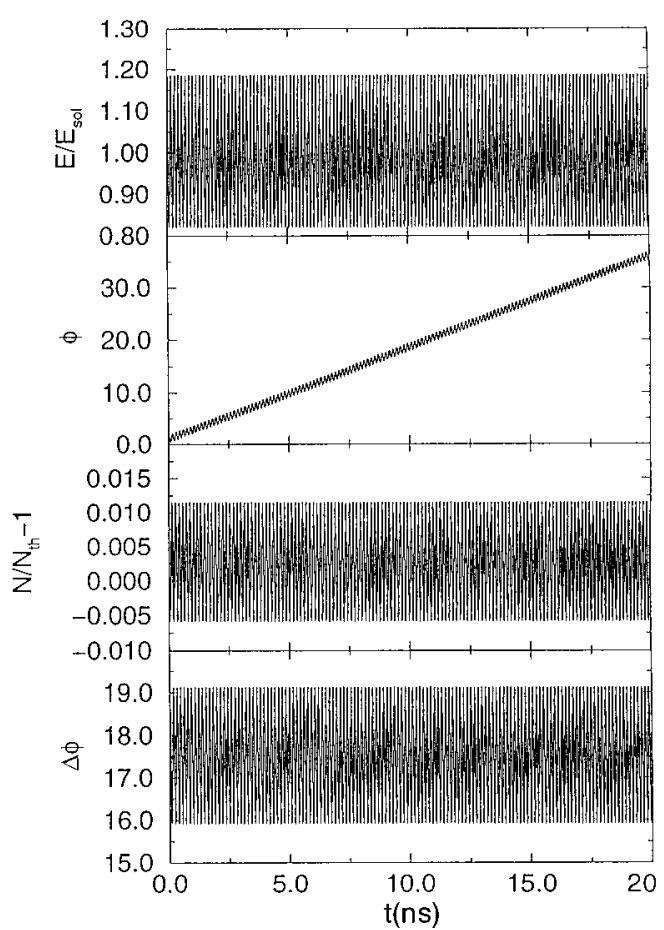

FIG. 7. (a) Three-dimensional plot of the torus developed from the external-cavity mode $i=-1$ in the $\left(\Delta \phi, N / N_{\text {th }}-1, E / E_{\text {sol }}\right)$ space and time evolution of the laser variables $E(t), \phi(t), N(t)$, and $\Delta \phi$. Same parameters as Fig. 6 . In the three-dimensional plot only one round-trip is shown, while in the time series two round-trips are shown. (b) As in (a) but of the limit cycle developed from the external-cavity mode $i=3$. (c) As in (a) but of the torus developed from the external-cavity mode $i=6$.

In Fig. 7 we show some of these attractors in more detail. Figure 7(a) shows the torus developed from mode $i=-1$, Fig. 7(b) the limit cycle developed from mode $i=3$, and Fig. 7 (c) the torus developed from mode $i=6$. We show the attractor in the $\left(\Delta \phi, N / N_{\mathrm{th}}-1, E / E_{\mathrm{sol}}\right)$ space (the circle indicates the location of the destabilized external-cavity modes, and the crosses indicate the nearby antimodes), and the time evolution of $E, \phi, N$, and $\Delta \phi$.
Clearly, the dynamics in each of the quasiperiodic tori has two different stages. The first stage consists of large modulation. We shall call this the "relaxation-oscillation" stage. The modulation affects all of the laser variables (intensity, optical frequency, and carrier density), which oscillate with the same modulation frequency (which is very close to $f_{\mathrm{RO}}$ ). The oscillations are not large pulses in between power dropouts (as occurs in the low-frequency fluctuations regime near 

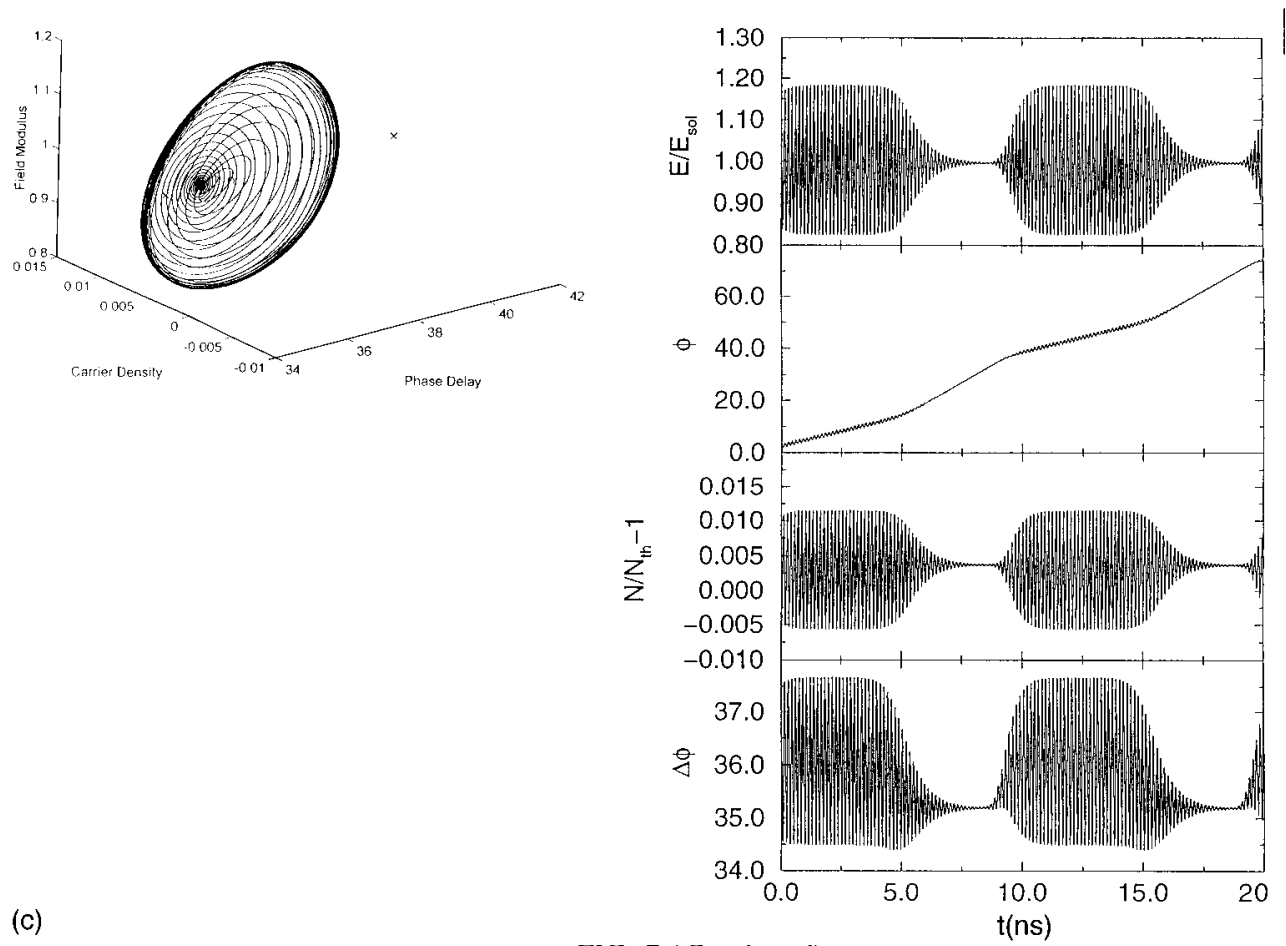

(c)

FIG. 7 (Continued).

the laser threshold) but moderate oscillations around the stationary state $E_{s, i}, N_{s, i}, \omega_{i} \tau$ given by Eqs. (4)-(6) (roughly speaking $\Delta E / E_{\mathrm{sol}} \leqslant 20 \%, \Delta N / N_{\mathrm{th}} \leqslant 1.5 \%$, and $\Delta \phi \leqslant \omega_{i} \tau$ $\pm 2.5)$. In the relaxation-oscillation stage, the laser has a mean optical frequency $\omega_{1}$ that is positive and is approximately the same for all the modes.

In the second stage, the laser evolves in the vicinity of the external-cavity-mode solution; the evolution is slow, the amplitude of the modulation markedly decreases, and the laser has a mean optical frequency $\omega_{2}$ that depends on the particular external-cavity mode. $\omega_{2}$ is negative in what we shall call a type-I torus, and positive in a type-II torus. In the phase space, the type-I tori are located between the fixed points and the limit cycles, while the type-II tori are located above the limit cycles (see Fig. 6).

For those ECMs whose $\omega_{i}$ is close to $\omega_{1}$, the limit cycles persist over the widest range of values of $\gamma$.

The type-I tori and the type-II tori are topologically distinct. In the type-I tori, two pseudo fixed points exist (located near the center of the tori). Between these pseudo fixed points the trajectory spirals in a channel (which is often extremely tiny) which connects the two sides of the torus (this is what we previously called the second stage of the evolution). Then, it evolves over the "outside" of the torus, in a "pseudo" limit cycle (this is what we previously called the first stage of the evolution) before going back to the center.

In the type-II tori, only one pseudo fixed point exists. The attractor resembles a cone (with the pseudo fixed point in its vertex), and the trajectory evolves over the "outside" of the cone (first stage), and then near the vertex (second stage).

The topological differences between the type-I and the type-II tori probably arise from the location of the unstable fixed points in the phase space. For the type-I tori (which develop from ECMs with not too large mode number $i$ ) the antimodes are located almost symmetrically with respect to the destabilized external-cavity mode from which the attrac- tor originated. For the type-II tori (which develop from ECM's with large, positive $i$ ) one antimode is very close to the destabilized external-cavity mode from which the attractor originated (see Fig. 1).

There is a kind of phase matching condition (or "frequency locking', ) in the dynamics. We refer to frequency locking as the situation where the phase difference $\Delta \phi(t)$ $=\phi(t)-\phi(t-\tau)$ oscillates periodically with mean value close to the stationary value $\left(\omega_{i}-\omega_{0}\right) \tau$. We see in Fig. 7(a) that for the quasiperiodic torus $\Delta \phi$ oscillates almost periodically for a while (with frequency $\approx f_{\mathrm{RO}}$ ), but since the second frequency involved in the dynamics is not $1 / \tau$ but is slightly lower, at a certain point $\Delta \phi$ becomes too large, the phase matching breaks, and the laser adjusts by evolving close to the unstable external-cavity-mode solution. Then, it initiates the mode locking process again. In type-II tori $\Delta \phi$ decreases before the mode locking breaks [Fig. 7(c)].

Figure 8 shows the optical spectrum of the three attractors shown in Fig. 7. In these spectra the positive frequencies close to $\omega_{1}$ have relatively important peaks, even though the torus of Fig. 7(a) develops from an external-cavity mode with $\omega_{i}<\omega_{1}$, and the torus of Fig. 7(c) from an externalcavity mode with $\omega_{i}>\omega_{1}$.

The type of attractor which develops from a particular external-cavity mode depends on the location of the mode in the $(\omega, \gamma)$ plane. In this plane, two curves are important. The first is the curve where saddle-node bifurcations occur and pairs of fixed points are born, and the second is the curve where Hopf bifurcations occur and the stable modes become limit cycles.

A saddle-node bifurcation occurs when

$$
f(\omega)=\frac{d f(\omega)}{d \omega}=0 .
$$



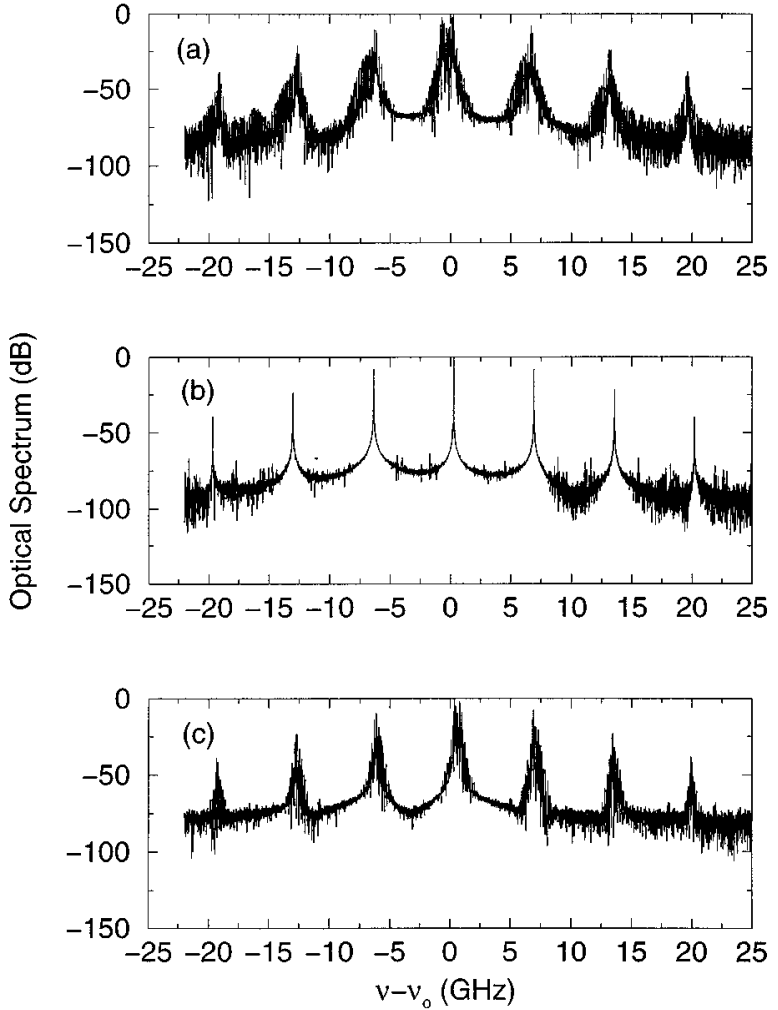

FIG. 8. Optical spectrum of the attractors shown in Fig. 7.

Therefore the critical feedback level at which a pair of modes of frequency $\omega_{i}^{s n}$ appears is

$$
\gamma_{s n}=\frac{-1}{\tau \sqrt{1+\alpha^{2}} \cos \left[\omega_{i}^{s n} \tau+\arctan (\alpha)\right]},
$$

where $\omega_{i}^{s n}$ is a solution of

$$
\omega_{i} \tau-\omega_{0} \tau=\tan \left[\omega_{i} \tau+\arctan (\alpha)\right]
$$

(The set of $\omega_{i}^{s n} \tau$ values depends only on the value of $\alpha$ and for large mode number they are separated by approximately $2 \pi$.)

The feedback level above which a Hopf bifurcation occurs and mode $i$ becomes unstable is [36]

$$
\gamma_{H}=\frac{-2 \lambda_{R}}{\sqrt{1+\alpha^{2}} \cos \left[\omega_{i} \tau-\arctan (\alpha)\right]\left[1-\cos \left(\omega_{R} \tau\right)\right]},
$$

where

$$
\lambda_{R}=\frac{1}{2}\left(\frac{1}{\tau_{s}}+G_{N} E_{\mathrm{sol}}^{2}+\frac{E_{\mathrm{sol}}^{2}}{\tau_{p}} \varepsilon\right)
$$

is the damping rate of the solitary laser, $\omega_{R}=2 \pi f_{\mathrm{RO}}$ $=\sqrt{G_{N}\left(J-J_{\text {th }}\right)}$, and $\omega_{i}$ is a solution of Eq. (4). If $\omega_{R} \tau$ $\approx(2 n+1) \pi$, a good approximation of Eq. (11) is

$$
\gamma_{H}=\frac{-\lambda_{R}}{\sqrt{1+\alpha^{2}} \cos \left[\omega_{i} \tau-\arctan (\alpha)\right]} .
$$

In Fig. 9 we show the location of the saddle-node and Hopf curves in the $(\omega \tau, \gamma)$ plane, for three different time delays, and $\omega_{0} \tau$ fixed. (Obviously the Hopf curve is located above the saddle-node curve.) The saddle-node and Hopf curves are similar to hyperbole. The Hopf curve has its minimum shifted towards the modes with mode number $i>0$ (a shift that increases as $\tau$ increases), while the saddle-node curve is symmetric with respect to the solitary laser mode $\left(i=0, \omega_{0} \tau=6 \mathrm{rad}\right)$.

The two curves are almost parallel in the $\omega \tau<\omega_{0} \tau$ side of the plane, and are tangent at some point in the $\omega \tau>\omega_{0} \tau$ side of the plane. The relative positions of these two curves in the $(\omega \tau, \gamma)$ plane leads to the existence, for a given value of $\gamma$ above the minimum for any Hopf bifurcation, of four categories of attractors: (1) The stable modes with mode number $i<0$ which have not undergone the Hopf bifurcation yet (for these modes, $\left.\gamma_{H}>\gamma\right)$; the maximum gain mode and its neighboring modes belong to this category, as discussed by Levine et al. in [37]; (2) the modes situated to the left of the minimum of the Hopf curve, which have $\gamma_{H}<\gamma$ (they have undergone a Hopf bifurcation); these modes rapidly yield to tori of type I; (3) the modes situated on the bottom of the Hopf curve, which usually yield to limit cycles for a very large feedback range; these attractors are the most stable, i.e., they are the last to lose stability and the first to merge; (4) the modes situated near but below the region where the saddlenode and Hopf curves become tangent. These modes yield to limit cycles for a small range of $\gamma$ but with increasing feedback they turn into tori of type II and then lose stability.
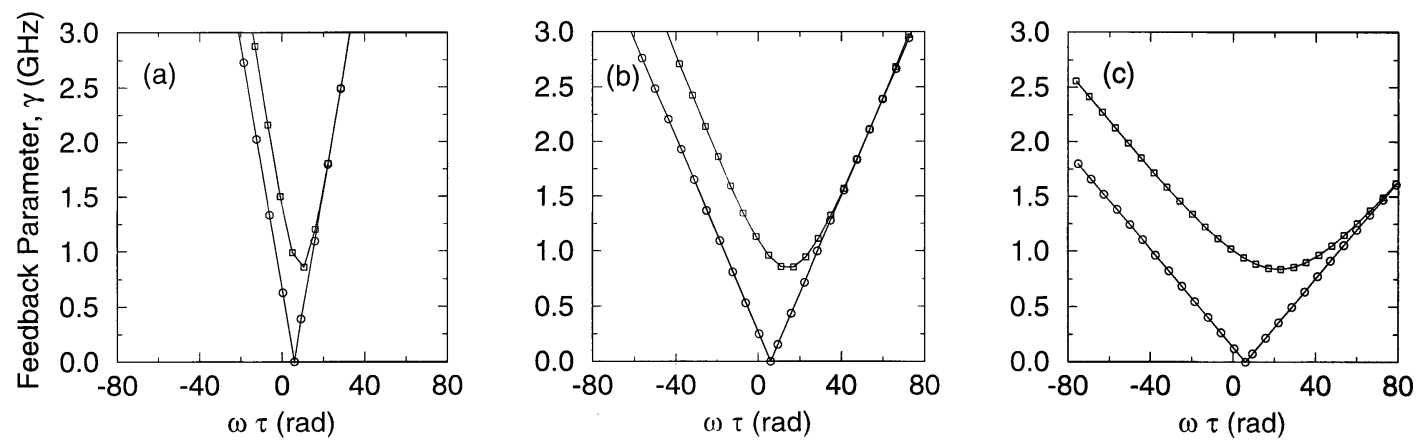

FIG. 9. Saddle-node $(\bigcirc)$ and Hopf $(\square)$ curves for $\gamma=1.2 \times 10^{9} \mathrm{~s}^{-1}$, and (a) $\tau=2 \mathrm{~ns}$, (b) $\tau=5 \mathrm{~ns}$, (c) $\tau=10 \mathrm{~ns}$. 
For the ECMs above the region where the saddle-node and Hopf curves become tangent we could not find any stable attractors (neither steady state, periodic, nor chaotic attractor). This region corresponds to modes with high positive mode number, which exist only for high feedback levels. Trajectories starting from initial conditions in these modes leave and end up on the already merged attractors.

By contrast, for the ECMs with mode number $i \ll 0$ (which are also only created at high feedback levels), numerical simulations clearly show that they remain stable fixed points until the Hopf bifurcation occurs.

Figure 9 offers an obvious analogy to the behavior of a semiconductor laser with external injection (see, e.g., Fig. 1 of [38]). The frequency symmetry of the steady states and the Hopf bifurcation threshold with a shifted minimum frequency are strikingly similar.

Conditions for the stability of the single ECM solutions cannot be determined analytically because the characteristic equation is a complicated transcendental equation for the complex growth rate of small perturbations. Based on our numerical results, we speculate that the mode and antimode fixed points with $i \gg 0$ are both unstable from the beginning. Similar behavior typically occurs near a saddle-node-Hopf codimension two bifurcation point (on one side of the bifurcation point there is a supercritical saddle node followed by a supercritical Hopf bifurcation, and on the other side, a subcritical saddle node followed by a subcritical Hopf bifurcation [39]).

Finally, let us discuss the effects of the relevant parameters on the dynamics. Two important parameters are the pump current $J$ and the gain saturation coefficient $\varepsilon$. An increase of $J$ or $\varepsilon$ increases the dissipation, and diminishes the size of the ECM attractor (the two distinct stages of the dynamics that we described before do not exist, or become less distinct, if either the laser is biased close to threshold or the nonlinear gain is neglected). Increasing $\alpha$ or $\gamma$ increases the instabilities and the size of the ECM attractor. An increase of $\tau$ leaves the attractor size relatively unchanged, but the "tunnel"' in the center of the torus becomes more narrow (this behavior occurs for long delay times, since for short $\tau$ these attractors are either fixed points or limit cycles). There are also very narrow regions of the delay time in which a kind of frequency locking occurs (between $f_{\text {ext }} \approx 1 / \tau$ and $f_{\mathrm{RO}}$ ), and the topological structure of the attractor changes abruptly (for instance, it becomes a fixed point).

\section{SUMMARY AND CONCLUSIONS}

We have studied in detail the dynamics of a single-mode laser diode biased far from threshold and subjected to moderately strong feedback from a distant reflector. In the hopping dynamics (at the beginning of the merging of the attractors developed from the ECMs, which corresponds to the beginning of regime IV in the classification of the externalcavity laser dynamics of [40]), not all the merged attractors are equally visited. The probability distribution of visiting single ECM attractors (when very few attractors have merged) is highly asymmetric and centered on a frequency slightly larger than $\omega_{0}$. This leads to a positive frequency shift in the spectrum of the laser. For increasing feedback, as more attractors merge, the probability distribution becomes

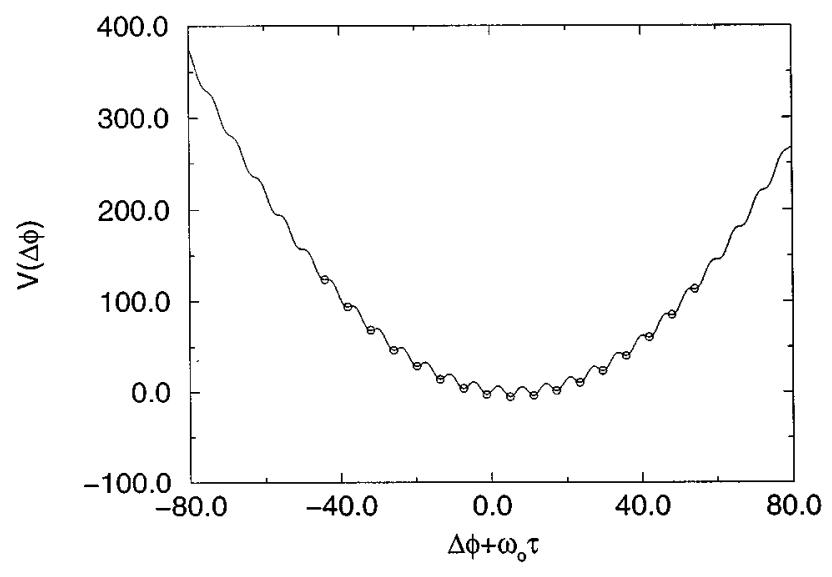

FIG. 10. Potential of Eq. (13) for the same parameter values as Fig. 1. The circles indicate the position of the destabilized modes.

symmetric and more nearly centered on $\omega_{0}$, and the frequency shift gradually disappears. In this regime (which corresponds to moderately strong feedback levels) the modes with large positive frequency shift with respect to the solitary laser frequency are highly unstable, and the modes with large negative frequency shift have very narrow basins of attraction. The laser is very rarely observed to operate in the vicinity of these modes. The different stability properties of the ECM attractors can be understood from the dynamics of the laser when it operates in a single ECM attractor. The dynamics consist of two clearly distinguished stages. There are large amplitude fast oscillations of the laser variables, and there is slow evolution (close to the external-cavity steady state) in which the amplitude of the oscillations is markedly decreased. While the mean emission frequency $\omega_{2}$ in the second stage depends on the particular ECM, the average frequency $\omega_{1}$ in the first stage is positive and approximately the same for all the modes. The modes with $\omega_{i}$ close to $\omega_{1}$ are the most stable. They lead to attractors which are limit cycles over a large feedback range. The attractors developed from them are the last to lose stability, and the first to merge.

It has been shown that when the ECMs are all stable fixed points, the dynamics of the mode hopping driven by spontaneous emission noise is governed by a potential model, for which the derived transition times are in surprisingly good agreement with experiments [41-43]. In this model the intensity of the light is assumed constant, which leaves the phase of the electric field as the only independent variable of the system. According to this model, the dynamics is governed by the potential

$$
V(\Delta \phi)=\Delta^{2} \phi / 2 \tau-\gamma \sqrt{1+\alpha^{2}} \cos \left(\Delta \phi+\omega_{0} \tau+\arctan \alpha\right) .
$$

Figure 10 shows $V(\Delta \phi)$ for the same parameter values as Fig. 1. The modes and antimodes are located, respectively, in the local potential minima and maxima. In the potential of Eq. (13), the external-cavity mode with deepest valley is also the mode with narrowest minima. Since the linewidth of a specific mode is inversely proportional to the square of the potential curvature at that point, the dominant mode is the mode with minimum linewidth (which is also the mode for which the frequency $\omega_{i}$ is closest to $\left.\omega_{0}\right)[41,42]$. In [44] the inclusion of excited relaxation oscillations in the potential 
picture leads to a nonlocal potential, which improved the predictions of the "old" model.

While the potential of Eq. (13) gives a reasonable description of the steady-state properties and dynamics on long time scales (for low feedback levels), it fails to describe the transient switch on of the laser. In [45] a time-dependent potential was proposed but it was found that it leads to an incorrect prediction of the final state selected.

Therefore, in terms of the phenomenological classification of Tkach and Chraplyvy [40], the potential picture applies to the stationary behavior in regimes I, II, and part of III. Our results suggest the existence of an "effective potential" that applies to the dynamical behavior in regimes III and beginning of IV, which has roughly the shape of the Hopf curve, i.e., it has its minimum shifted towards higher frequencies.

\section{ACKNOWLEDGMENTS}

This work was supported by Project No. 47 of the BIDCONICYT, the Comision Sectorial de Investigacion Cientifica (CSIC), and the PEDECIBA.
[1] D. Lenstra, B. H. Verbeek, and A. J. den Boef, IEEE J. Quantum Electron. QE-21, 674 (1985).

[2] J. Mørk, B. Tromborg, and J. Mark, IEEE J. Quantum Electron. 28, 93 (1992).

[3] K. Petermann, IEEE J. Sel. Top. Quantum Electron. 1, 480 (1995).

[4] G. H. M. van Tartwijk and D. Lenstra, Quantum Semiclassic. Opt. 7, 87 (1995).

[5] A. T. Ryan and G. P. Agrawal, IEEE J. Quantum Electron. 30, 668 (1994).

[6] B. W. Liby and D. Statman, IEEE J. Quantum Electron. 32, 835 (1996).

[7] N. Kikuchi, Y. Liu, and J. Ohtsubo, IEEE J. Quantum Electron. 33, 56 (1997).

[8] S. I. Turovets, J. Dellunde, and K. A. Shore, J. Opt. Soc. Am. B 14, 200 (1997).

[9] J. Wieland, C. R. Mirasso, and D. Lenstra, Opt. Lett. 22, 469 (1997).

[10] Y. Kitaoka, H. Sato, K. Mizuuchi, K. Yamamoto, and M. Kato, IEEE J. Quantum Electron. 32, 822 (1996).

[11] P. Kürz and T. Mukai, Opt. Lett. 21, 1369 (1996).

[12] C. R. Mirasso, P. Colet, and P. Garcia-Fernandez, IEEE Photonics Technol. Lett. 8, 299 (1996).

[13] V. Annovazzi-Lodi, S. Donati, and A. Scirè, IEEE J. Quantum Electron. 33, 953 (1996).

[14] J. Martin-Regalado, G. H. M. van Tartwijk, S. Balle, and M. San Miguel, Phys. Rev. A 54, 5386 (1996).

[15] M. Homar, J. V. Moloney, and M. San Miguel, IEEE J. Quantum Electron. 32, 553 (1996).

[16] J. Dellunde, M. C. Torrent, C. R. Mirasso, E. HernandezGarcia, and J. M. Sancho, Opt. Commun. 115, 523 (1995).

[17] G. H. M. van Tartwijk and M. San Miguel, IEEE J. Quantum Electron. 32, 1191 (1996).

[18] R. Lang and K. Kobayashi, IEEE J. Quantum Electron. QE16, 347 (1980).

[19] J. Mørk, B. Tromborg, and P. L. Christiansen, IEEE J. Quantum Electron. 24, 123 (1988).

[20] A. Hohl, H. J. C. van der Linden, and R. Roy, Opt. Lett. 20, 2396 (1995).

[21] C. H. Henry and R. F. Kazarinov, IEEE J. Quantum Electron. QE-22, 294 (1986).

[22] T. Sano, Phys. Rev. A 50, 2719 (1994).
[23] G. H. M. van Tarwijk, A. M. Levine, and D. Lenstra, IEEE J. Sel. Top. Quantum Electron. 1, 466 (1995).

[24] I. Fischer, G. H. M. van Tartwijk, A. M. Levine, W. Elsässer, E. Göbel, and D. Lenstra, Phys. Rev. Lett. 76, 220 (1996).

[25] M. Giudici, C. Green, G. Giacomelli, U. Nespolo, and J. R. Tredicce (unpublished).

[26] G. Huyet, S. Balle, M. Giudici, C. Green, G. Giacomelli, and J. R. Tredicce (unpublished); G. Huyet, S. Hegarty, M. Giudici, B. de Bruyn, and J. G. McInerney (unpublished).

[27] M. Pan, B. Shi, and G. R. Gray, Opt. Lett. 22, 166 (1997).

[28] C. Masoller, Phys. Rev. A 50, 2569 (1994).

[29] T. Hirono, T. Kurosaki, and M. Fukuda, IEEE J. Quantum Electron. 32, 829 (1996).

[30] J. E. Bowers, Solid-State Electron. 30, 1 (1987).

[31] M. Willatzen, A. Uskov, J. Mørk, H. Olesen, B. Tromborg, and A. P. Jauho, IEEE Photonics Technol. Lett. 3, 606 (1991).

[32] H. Haug and H. Haken, Z. Phys. 204, 262 (1967).

[33] C. H. Henry, IEEE J. Quantum Electron. QE-18, 259 (1982).

[34] C. Masoller, C. Cabeza, and A. Sicardi Schifino, IEEE J. Quantum Electron. 31, 1022 (1995).

[35] G. P. Agrawal and N. K. Dutta, Long-Wavelength Semiconductor Lasers (Van Nostrand Reinhold, New York, 1986).

[36] A. Ritter and H. Haug, J. Opt. Soc. Am. B 10, 130 (1993); 10, 145 (1993).

[37] A. M. Levine, G. H. M. van Tartwijk, D. Lenstra, and T. Erneux, Phys. Rev. A 52, R3436 (1995).

[38] W. A. van der Graaf, A. M. Levine, and D. Lenstra, IEEE J. Quantum Electron. 33, 434 (1997).

[39] J. Guckenheimer and P. Holmes, Nonlinear Oscillations, Dynamical Systems, and Bifurcations of Vector Fields (SpringerVerlag, New York, 1983).

[40] R. W. Tkach and A. R. Chraplyvy, IEEE J. Lightwave Technol. LT-4, 1655 (1986).

[41] J. Mørk and B. Tromborg, IEEE Photonics Technol. Lett. 2, 21 (1990).

[42] J. Mørk, M. Semkow, and B. Tromborg, Electron. Lett. 26, 609 (1990).

[43] D. Lenstra, Opt. Commun. 81, 209 (1991).

[44] G. H. M. van Tartwijk and D. Lenstra, Phys. Rev. A 50, R2837 (1994).

[45] E. Hernandez-Garcia, N. B. Abraham, M. San Miguel, and F. De Pasquale, J. Appl. Phys. 72, 1225 (1992). 